\title{
The Age of Theranostics in Nuclear Medicine: Bangladesh Standpoint
}

\author{
Lutfun Nisa and Shamim Momtaz Ferdousi Begum National Institute of Nuclear Medicine \& Allied \\ Sciences, Dhaka Correspondence Address: Professor Lutfun Nisa. Email nisa.lutfun@gmail.com
}

Theranostics is coupled diagnostic imaging and targeted therapy at the molecular and cellular level with a single agent. The earliest and best known element in theranostic is radioactive iodine. Beginning in the 1940s this amazing radionuclide has revolutionized the treatment of hyperthyroidism and differentiated thyroid carcinoma all over the world. In Bangladesh too the foundation of nuclear medicine was laid around radioactive iodine and the thyroid gland and still now continues to thrive on it.

A throwback in history gives us the outstanding story of radioiodine therapy initiated by Dr. Saul Hertz in 1941, who was the first physician to use this radioisotope to treat diffuse toxic goiter (1). Then in 1943, Dr. Samuel Seidlin used it to treat patients with functioning differentiated thyroid cancer metastases (2). Decades later radioiodine therapy in thyroid disease still remains as strong as ever with added innovative adjuncts and multiple new approaches. Based on this original model, many new exciting radiopharmaceuticals have emerged in the management of tumours in oncology. This reproduction of integrated tumour diagnosis and therapy is given a brand new label called Theranostic and/or theragnostics which is a term coined in 2002 by the CEO of PharmaNetics, John Funkhouser (3). Theranostics emphasizes the existence of successful therapy using radionuclides and radiopharmaceuticals (4).

Nuclear medicine imaging plays a critical role in theranostics since the visualization of potential targets can help predict if a particular patient would benefit from such an approach. Both gamma and positron emitters $(\beta+)$ are involved in nuclear medicine imaging. Gamma emitters, such as technetium-99m (99mTc) or iodine-123 (123I), can be located using gamma cameras (planar imaging) or SPECT (single photon emission computed tomography) (5). Positron emission tomography (PET) gives better resolution using positron emitters, such as gallium- 68 and fluorine-18 (6). Additionally the introduction of hybrid technology: positron emission tomography/computed tomography (PET-CT) and single-photon emission computed tomography (SPECT)/CT gives precise functional imaging with exquisite anatomical details. Consequently medical imaging has been transformed by hybrid technology and plays a significantly renewed role in theranostics where a particular isotope is used for imaging and a similar radioisotope which is either radiolabeled differently or given in different dosages is used for therapy. Radioisotope of iodine is a case in point, where ${ }_{131} \mathrm{I}$ is both a beta and gamma emitter while 123I which is a different isotope of same element is a gamma emitter. Thus 123I is used to visualize the functioning thyroid cells through imaging while ${ }_{131} \mathrm{I}$ is used to destroy those same thyroid cells. Another example is iodine-131 and lutetium-177 both of which are gamma and beta emitters; thus, these agents can be used for both imaging and therapy (7).

Nuclear targeted therapies play an essential role, especially in patients with advanced neuroendocrine tumors, such as gastroenteropancreatic (GEP) tumors, bronchopulmonary neuroendocrine tumors, pheochromocytoma, and neuroblastoma $(8,9)$.

Two commonly used somatostatin analogs DOTATOC and DOTATATE are labelled with Lu-177 or Y-90 for therapy in neuroendocrine tumors and the therapy response can be evaluated with accompanying nuclear imaging using SPECT (Lu-177) or PET (Y-90) (10). 
A more contemporary theranostic agent is the prostate-specific membrane antigen inhibitor, PSMA617 , that targets a cell surface protein on prostate cancer cells. When PSMA-617 is radiolabeled with gallium-68 (a diagnostic imaging agent) and lutetium-177 (a therapeutic agent), it becomes both a very specific diagnostic and therapeutic agent, and follow-up studies using this agent on patients with prostate cancer reveal a very positive response to therapy (11).

As of now established theranostics are Lutetium PSMA therapy for metastatic or treatment-resistant prostate cancer; Yttrium-90 SIRT therapy for liver cancer; Iodine-131 therapy for thyrotoxicosis and thyroid cancer; Radium-223 therapy for metastatic prostate cancer in bones and Yttrium-90 radiosynovectomy therapy for inflammatory synovitis of joints.

Advances in the understanding of cancer biology, developments in hybrid imaging technologies and expansion of therapeutic options have all contributed to the concept of patient- centered cancer care (12). However an important consideration in theranostics is the potential for high cumulative doses of radioactive agents after multiple repeated cycles. Dosimetric evaluation, monitoring and preventive measures to curb toxicity are therefore matters of utmost importance in theranostics.

Many new radiopharmaceutical targets that are capable of tumour characterisation, angiogenesis, proliferation, apoptosis and receptor expression continue to be developed. In addition nuclear theranostics is broadening its narrow sense of guiding the radionuclide therapy of cancers to the imageguided therapy of other non-communicable diseases such as carotid or coronary artery stenosis (13).

Overall the application of precision medicine not only promises to enhance the life of patients and increase the quality of clinical practice and targeted care pathways, but also to lower overall healthcare costs through early-detection, prevention, accurate risk assessments and efficiencies in care delivery(14).
Personalized medicine therefore appears to be very promising in contributing to the overall health issue of a population. Nevertheless the establishment of theranostics and precision medicine in a country with dearth of 'state-of the art' laboratory settings is not an easy task. As always, the transfer of technological innovations from an advanced laboratory to a less developed one is a competitive one. The question is whether Bangladesh can effectively face up to this challenge. First and foremost a well equipped laboratory both for imaging and microbiology are absolute necessities. While the Nuclear Medicine institute in Bangladesh has the infrastructure for PET-CT Cyclotron technology, it does not have a molecular microbiology laboratory within the same organization. Establishment of a molecular microbiology facility within a conventional microbiology laboratory appears critical. A regulatory arrangement to initiate the collaboration of different kinds of specialist and hybrid setting is essential for high quality standard performance.

Moreover fundamental issues that are in compliance with good laboratory practice must be addressed. These would include appropriate laboratory administration, a well-designed facility, laboratory procedure standardization, waste management system, code of practice, equipment installation and laboratory personnel training. Beyond laboratory standardization, the interfacing of different specialists for multi-skilled endeavours is a key issue for development of the field. As noted by S. Jakka et al, theranostics is an approach considered as "P4 medicine," that is predictive, preventive, personalized, and participatory (14). In fact, personalized medicine may be considered an extension of traditional approaches to understanding and treating disease but with greater precision (15).

In conclusion development of molecular targeting therapy is a challenge that requires major undertaking. A motivated and dedicated team committed to work towards obtaining legislation, convincing the government for logistic support, initiating multispecialist cooperation and international expert 
assistance can help to implement the project. Clinical awareness of theranostics as a precision medicine, medical education reforms, adoption of theranostics into research and clinical practice are some of the factors that are instrumental to achieve success. When all of the pieces of the infrastructure fall into place, theranostics can make a huge difference in the approach towards cancer and other diseases by eliminating unnecessary and ineffective therapies for more precise personalized therapy.

\section{REFERENCES}

1. Fahey FH, Grant FD, Thrall JH. Saul Hertz, MD, and the birth of radionuclide therapy. EJNMMI Phys. 2017;4(1):15.

2. Siegel E. The beginnings of radioiodine therapy of metastatic thyroid carcinoma: a memoir of Samuel M. Seidlin, M. D. (1895-1955) and his celebrated patient. Cancer Biother Radiopharm. 1999;14(2):71-9

3. Jeelani S, Reddy RC, Maheswaran T, Asokan GS, Dany A, Anand B. Theranostics: A treasured tailor for tomorrow. J Pharm Bioallied Sci 2014;6(Suppl 1):S6-8.

4. Lee, D.S. \& Cheon, G.J. Nucl Med Mol Imaging (2019). https://doi.org/10.1007/s13139-019-00573-2

5. Holman BL, Tumeh SS. Single-photon emission computed tomography (SPECT): applications and potential. JAMA 1990;263(4):561-564. [PubMed]
6. Lammertsma AA. Forward to the Past: The Case for Quantitative PET Imaging J Nucl Med 2017; 58 (7): 10191024

7. Yordanova, Anna et al. "Theranostics in nuclear medicine practice"OncoTargets and therapy vol. 10 4821-4828. 3 Oct. 2017, doi:10.2147/OTT.S140671

8. Yao JC, Lagunes DR, Kulke MH. Targeted therapies in neuroendocrine tumors (NET): clinical trial challenges and lessons learned. Oncologist. 2013;18(5):525-32.

9. Oladejo AO. Gastroenteropancreatic neuroendocrine tumors (GEP-NETs) Approach to diagnosis and management. Ann Ib Postgrad Med. 2009;7(2):29-33.

10. Lee DS. From Nuclear Medicine to Nuclear Medicine Theranostics. Nucl Med Mol Imaging. 2015;49(2):83-84

11. Rahbar K, Afshar-Oromieh A, Jadvar H, Ahmadzadehfar H. PSMA Theranostics: Current Status and Future Directions. Mol Imaging 2018;17:1-9.

12. Hossein Jadvar, Xiaoyuan Chen, Weibo Cai, Umar Mahmood, Radiotheranostics in Cancer Diagnosis and Management. Radiology. 2018;286 (2): 388-400.

13. Dong Soo Lee1Nuclear Medicine and Molecular Imaging 2018;52:87-88 https://doi.org/10.1007/s13139-018-0519-8 Message from the Editor-in-Chief: Theranostic Imaging in 2018

14. Jakka S, Rossbach M. An economic perspective on personalized medicine. Hugo J. 2013;7(1):1-6.

15. Vogenberg FR, Isaacson Barash C, Pursel M. Personalized medicine: part 1: evolution and development into theranostics. P T. 2010;35(10):560-576. 